\title{
655 LANDSCAPE OF HELPER AND REGULATORY CD4+ T CELLS IN MELANOMA
}

${ }^{1}$ Giacomo Oliveira*, 'Kari Stromhaug, ${ }^{2}$ Susan Klaeger, ${ }^{1}$ Nicoletta Cieri, ${ }^{1}$ Bryan lorgulescu, ${ }^{1}$ Shuqiang Li, 'David Braun, ${ }^{1}$ Donna Neuberg, ${ }^{2}$ Steven Carr, ${ }^{1}$ Kenneth Livak, ${ }^{3}$ Dennie Tompers, ${ }^{1}$ Edward Fritsch, ${ }^{4}$ Megan Wind-Rotolo, ${ }^{2}$ Nir Hacohen, ${ }^{3}$ Moshe SadeFeldman, ${ }^{1}$ Derin Keskin, ${ }^{1}$ Patrick Ott, ${ }^{5}$ Scott Rodig, ${ }^{3}$ Genevive Boland, ${ }^{1}$ Catherine Wu. ${ }^{1}$ Dana-Farber Cancer Institute, Boston, United States; ${ }^{2}$ Broad Institute of MIT and Harvard, Cambridge, United States; ${ }^{3}$ Massachusetts General Hospital, Boston, United States; ${ }^{4}$ BristolMyers Squibb, Princeton, NJ, United States; ${ }^{5}$ Brigham and Women's Hospital, Boston, MA, United States

Background Within the tumor microenvironment, distinct CD4 $+\mathrm{T}$ cell subsets can play different and even opposite roles either promoting or suppressing anti-tumor responses through the recognition of antigens presented by human leukocyte antigen (HLA) class II molecules. However, how cancers coopt these processes to shape the intratumoral CD4+ landscape and achieve immune evasion remains incompletely understood. Methods We performed single-cell characterization of CD4+ tumor infiltrating lymphocytes (TILs) collected from four human melanoma with low or high HLA-class II expression and we utilized TCR reconstruction and antigen specificity screening to unambiguously discover the tumor reactivity of CD4+ TILs. By testing TCR-transduced T cells against autologous patient-derived melanoma cell lines or against autologous antigen presenting cells (APCs) loaded with tumor lysates, we assessed the capacity of CD4+ TCRs to directly or indirectly recognize tumor cells. We defined the antigen-specificity of antitumor CD4+ TCRs by assessing their reactivity towards personal neoantigens (NeoAg) or public melanoma associated antigens (MAAs). Finally, we correlated NeoAg burden and HLA-class II expression in a series of 116 melanoma specimens from 4 independent cohorts of patients.

Results Analysis of single-cell data showed that the cluster distribution of cells within each CD4+ TCR clonotype family was highly homogeneous and appeared to follow 3 distinct major phenotypes, corresponding to non-exhausted memory cells, exhausted cells and regulatory cells $\left(\mathrm{T}_{\text {Regs }}\right)$. Strikingly, clonally expanded CD4 $+\mathrm{T}_{\mathrm{Reg}}$-TILs were highly abundant within the tumor microenvironment of HLA class II $^{\text {pos }}$ melanomas. We found that TCRs from exhausted cytotoxic CD4+ $\mathrm{T}$ cells could be directly triggered by melanoma cells not only through recognition of HLA class II restricted antigens, but also through presentation of HLA class I restricted MAAs. $\mathrm{T}_{\text {Reg }}$-TCRs could be indirectly elicited through presentation of tumor antigens via APCs. Notably, numerous tumor-reactive CD4 $+\mathrm{T}_{\mathrm{Reg}}$-TCRs were directly stimulated by HLA class II $^{\text {pos }}$ melanoma and demonstrated specificity for melanoma NeoAgs. In HLA class II $^{\text {pos }}$ melanomas, the clonal expansion of numerous tumor-reactive and NeoAg-specific $\mathrm{T}_{\text {Regs }}$-clones appeared to be favored by a dramatically high tumor NeoAg load. Analysis of 116 melanoma specimens confirmed the association of elevated HLA-class II expression with extremely high $\mathrm{NeoAg}$ burden.

Conclusions Our data elucidate the landscape of infiltrating $\mathrm{CD} 4+\mathrm{T}$ cells in melanoma and point to presentation of HLA-class II restricted NeoAgs and direct engagement of immunosuppressive $\mathrm{CD} 4+\mathrm{T}_{\mathrm{Regs}}$ as a novel mechanism of immune evasion favored in HLA class II $^{\text {pos }}$ melanoma.

http://dx.doi.org/10.1136/jitc-2021-SITC2021.655 